\title{
Monte Carlo-Alternative Probabilistic Simulations for Analog Systems
}

\author{
Rasit Onur Topaloglu \\ University of California at San Diego \\ Computer Science and Engineering Department \\ La Jolla, CA, 92093 \\ rtopalog@cse.ucsd.edu
}

\begin{abstract}
Probabilistic system simulations for analog circuits have traditionally been handled with Monte Carlo analysis. For a manufacturable design, fast and accurate simulations are necessary for time-to-market, design for manufacturability and yield concerns. In this paper, a fast and accurate probabilistic simulation alternative is proposed targeting the simulation of analog systems. The proposed method shows high accuracy for performance estimation combined with a 100fold reduction in run-time with respect to a 1000-sample Monte Carlo analysis.
\end{abstract}

\section{Introduction}

Probabilistic simulation for analog systems is a necessity due to increased process variations and mismatch in new technologies. Although probabilistic simulation has long been an interest, today's requirements necessitate faster and accurate simulation.

Most of the time, Gaussian densities for the output parameters are assumed [26]. Although Gaussian assumption might be sufficient for most input parameters, it is far from being accurate of an assumption for the output parameters for most analog blocks. Traditional techniques usually provide the mean and the variance for an output parameter. Yet, capturing the exact shape of a continuous density at the output is important for various considerations such as yield estimation.

Speed and accuracy of today's performance estimation techniques are lagging behind technology. Probabilistic simulation of large blocks brings significant time burdens on system designers. This has triggered us to come up with a technique to bring a solution to single input parameter probabilistic simulations. Since individual blocks are highly important in analog systems, single parameter simulations will give quite a deal of information. The whole system can then either be evaluated using single parameter as input and multiple parameters as output by selecting the dominant input parameter, or solving the system separately for more input parameters individually, followed by superposition, e.g. as usually done in sensitivity analysis [25]. The proposed estimation methods can be used for optimization, e.g. design parameter tuning, as well. Most multiparameter optimization techniques also work on single parameters at a time for improved convergence [20].

We first introduce the basis of the proposed technique, probability discretization and sample propagation implemented using a forward operation, where significant runtime improvement over Monte Carlo is gained through weight propagation and systematic sampling. Then, we propose the re-binning algorithm to convert the propagated samples to a continuous density. A traditional spline interpolation follows re-binning. Then we provide the performance estimation algorithm. We have used behavioral simulations on the jitter analysis of a phase-locked loop to validate the proposed computational technique.

\section{Previous Work}

A number of approaches for probabilistic simulation of analog blocks has been proposed. [12] and [9] have used principal component analysis on the correlation matrix of process variations to reduce the number of variables that are responsible for mismatch. In [23], sensitivity analysis followed by response surface polynomial fitting and Monte Carlo sampling are implemented. [29] has used regression analysis including second order terms. [8] has used variance propagation.

Particular interest in probabilistic simulation has been in the area of mismatch and process variation simulation. [21] has used Tailor series expansion. [6] has used sensitivity analysis and assumed that there are at least the same number of output parameters as there are input parameters, all of which are Gaussian. [17] has applied principal component analysis to account for correlations between input parameters. In [30], we have used hierarchical sensitivities for 
probabilistic simulation. These methods are good for calculating mean and variance. They will work accurate only if outputs are Gaussian assumption holds. Besides these, almost all designers count on Monte Carlo methods for probabilistic simulations. We have introduced a computationally expensive hierarchical version of the proposed method for probabilistic device simulations in [31].

Analog fault simulation is another field where probabilistic simulation has been important as analog faults are related to parameter probability densities. [19] has approximated mean and variances at the output analytically. [14] has used a hierarchical variance analysis. [24] and [32] have used a sensitivity-based Monte Carlo technique.

There are a number of Monte Carlo methods such as Latin Hypercube sampling [15] or rejection sampling [16] [3] [7]. Importance sampling and rejection sampling require a sampling density. As long as the sampling density is similar to the density to be estimated, these methods work fine. If such a sampling density is not available, a set of densities can be provided as in the Metropolis method. Yet, this requires more samples eventually. Gibbs sampling works for dimensions higher than 2, hence it is not applicable to the problem in this paper [16] [2].

Propagation of the densities is also a related topic. This can be done by Monte Carlo by propagating individual data points directly. Traditionally, variance propagation has been used. Parametric approaches, such as belief propagation [28], propagate certain moments of the density.

For behavioral simulations, particular attention has been on phase-locked loop simulations. [11] has introduced RF front-end models both for time and frequency domains. [18] has introduced a model calibration method for PLLs. [4] has compared numerical methods for phase/delay-locked systems. Behavioral simulations for phase-locked loops (PLLs) are important as most analyses require transient simulations over many cycles, indicating high run-times. The probabilistic nature makes the problem a couple orders harder.

Although probabilistic simulation has been traditionally used for analog circuits, digital circuits within the last couple of years took its portion [33] [13] [27] [34]. Recently, major contributions have come in the digital domain due to the necessity to have probabilistic static timing and leakage analysis. These methods are usually specific to the particular problem domain but not suitable for black-box type simulations. For example, only a limited set of all possible nonlinear operators, e.g. addition and maximum operations, are targeted in the probabilistic timing systems in the literature and the formulae that tie inputs to outputs are assumed to be analytically provided. RF domain performance estimation [1] and communication systems, on the other hand, are very similar to the analog system studied herein and hence can take advantage of the proposed techniques in this paper.

\section{Forward Discrete Probability Propagation}

In order to introduce FDPP, a number of definitions will be useful. Let $X$ be a random variable. We will denote the probability density of $X$ as $p d f(X) \cdot p d f(X)$ is assumed to be continuous. We propose to attain a discretization of this $p d f$ by sampling the $p d f$ at equidistant points of the random variable $X$.

\subsection{Density Discretization and Determin- istic Sampling}

The sampling can be done by dividing the $p d f(X)$ to bins and approximating the values that fall in any bin by the value at the mid-point of the bin. The bins are generated such that the highest and lowest samples are accounted for. Let $b_{i}$ be an enumeration over the bins where $1 \leq i \leq N$ and $N$ is the total number of bins. $b_{i}$ will be defined to be bounded by $[m+(i-1) \Delta, m+i \Delta)$, where $\Delta$ is the stepsize defined by $\frac{n-m}{N}$. The N'th bin, however, is bounded by $[m+(N-1) \Delta, m+N \Delta]$, which is equal to $[n-\Delta, n]$. We denote the sampled $p d f(X)$ as $\phi(X)$ or $\operatorname{spdf}(X)$.

The procedure of converting a $p d f$ to an spdf will be represented with the $\mathcal{Q}_{\mathcal{N}}$ operator:

$$
\phi(X)=\mathcal{Q}_{N}(p d f(X))
$$

The domain of this operator is a $p d f$, and the range of this operator is an $\phi(X)$. The result of this operator on the $p d f$ of random variable $X, \phi(X)$, is essentially a Riemann sum of impulses and is given by:

$$
\phi(X)=\sum_{i \in 1 . . . N} p_{i} \delta\left(x-w_{i}\right)
$$

where

$$
\begin{gathered}
p_{i}=\int_{m+(i-1) \Delta}^{m+i \Delta} p d f(X) d x \\
w_{i}=m+(i-1) \Delta+\frac{\Delta}{2}
\end{gathered}
$$

In these equations, $p_{i}$ corresponds to the probability that a sample of the random variable $X$ falls within the $i$ th bin $b_{i}$ and $v_{i}$ denotes the approximation of values of samples of $X$ within $b_{i}$. $w_{i}$ is the mid-point in the particular bin.

\subsection{Sample and Weight Propagation}

Assume that we have a random variable given as $X$ whose sampled $p d f$ is given by $\phi(X)$. Let $Y$ be another random variable that is given by a deterministic function $f$ of the given random variable: $Y=f(X)$. Then $\phi(Y)$ is given by the $\mathcal{F}$ operator as: 


$$
\phi(Y)=\mathcal{F}(\phi(X))
$$

which is defined as being equivalent to:

$$
\phi(Y)=\sum_{\forall s} p_{s}^{X} \delta\left(y-f\left(w_{s}^{X}\right)\right)
$$

Here, $Y$ is the output parameter to be observed and $X$ is the input parameter. The domain and the range of the $\mathcal{F}$ operator is an $\phi$. This operator presents a one-to-one relationship. There may be more than one $Y$ and $\mathcal{F}$, indicating the possibility of multiple outputs. The $\mathcal{F}$ operator essentially provides another sampled $p d f$, where the multiplication term $p_{s}^{X}$ denotes the probability at the point $f\left(w_{s}^{X}\right)$. Here, $s$ corresponds to the set of all samples belonging to the random variable $X$. An algorithm for the $\mathcal{F}$ operator is given below.

\section{Algorithm Implementing the $\mathcal{F}$ Operator:}

[1] While all samples of the target pdf computed:

[2] For each sample in $X$

[3] Place an impulse with height $p_{i}$ at $x=f\left(w_{i}\right)$

\subsection{Re-binning the Samples}

Certain calculations, such as yield estimation, require an accurate shape for the density function so that it can be integrated. But since $f$ is commonly a non-linear function, the samples may no longer be situated at fixed distances. Hence, forward propagation step should be followed by another binning process on the sampled probability density. Whenever an integral under the probability density is required, the shape of the density needs to be known accurately. To convert the FDPP samples into a density, spline interpolation is used on the samples. Interpolation requires equidistant samples. Hence, a re-bin operator needs to be implemented to separate out the samples evenly. Samples that fall in any particular bin will be approximated by a single impulse at the center of the corresponding bin, with the height of this impulse being the sum of the impulses that fall into this bin. Binning of impulses makes interpolation of the impulses possible. Without the binning process, neighboring impulses with largely differing sample probabilities would cause a great amount of noise. The re-bin operator is defined as:

$$
\phi^{\prime}(X)=\mathcal{R}_{N}(\phi(X))
$$

The re-bin operator can be implemented using the following algorithm:

Algorithm Implementing the $\mathcal{R}$ Operator:

[1] Find maximum and minimum values $v_{i}$ within impulses

[2] Divide this range into M bins

[3] For each bin

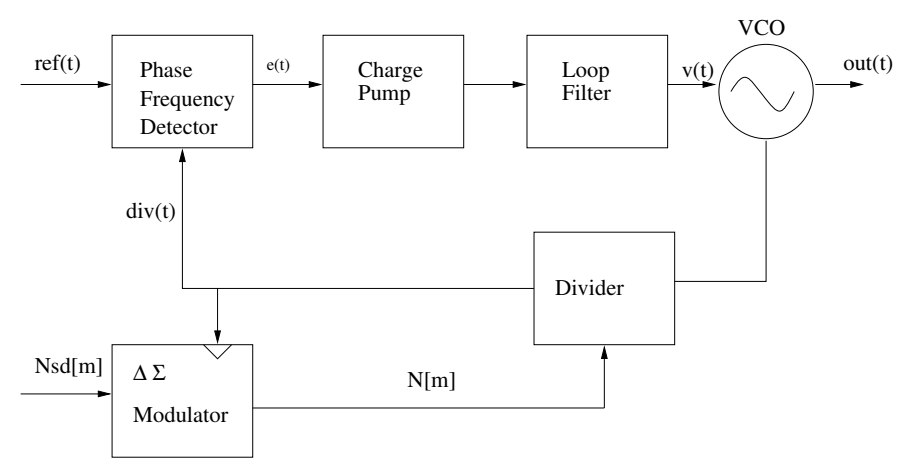

Figure 1. PLL architecture used in experiments

[4] Place a quantizing impulse at the center of the bin with a height $p_{i}$ equal to the sum of all impulses within the bin

The complexity of this algorithm is $O(n . m)$ where $m$ is the number of input samples and $n$ is the number of final samples. Since these numbers are rather small, the run-time is highly efficient in practice.

Notice that two different total bin numbers, $M$ and $N$, have been used although these are independent of the number of bins in the domain spdf. The choice of $M$ constitutes an initial binning, providing a fine resolution for the bin widths. $M$ is chosen larger than $N$ and the initial bin number.

The domain and range of the re-bin function is an spdf.

The $\mathcal{R}_{N}$ is defined as:

$$
\phi^{\prime \prime}(X)=\sum_{i} p_{i} \delta\left(x-w_{i}\right)
$$

where $p_{i}=\sum_{s_{j}} p_{j}$ such that $w_{j} \epsilon b_{i} . N$ in the $\mathcal{R}_{N}$ operator corresponds to the new number of bins, as the re-bin operator can bin the samples into a different number of bins than the one used in the $s p d f$ that this operation is applied to.

\section{Experimental Results}

Estimation of block parameter densities at the behavioral level can be useful when process variations need to be considered. Certain high-level analog blocks exhibit probability densities for their output parameters instead of a constant nominal value. For example, the loop filter in Figure 1 acquires a probability density for its output frequency as a result of process variations, as does the charge pump for the charged current. The deviation from a Gaussian density for both of these examples has been confirmed in the experiments we have conducted.

Phase-locked loops, being analog components, are an important part of almost all large-scale digital and mixedsignal systems. Their gate-accurate simulation takes very long time as transient analysis has to be done for many cycles. That's why, in this paper, we have chosen this block as 


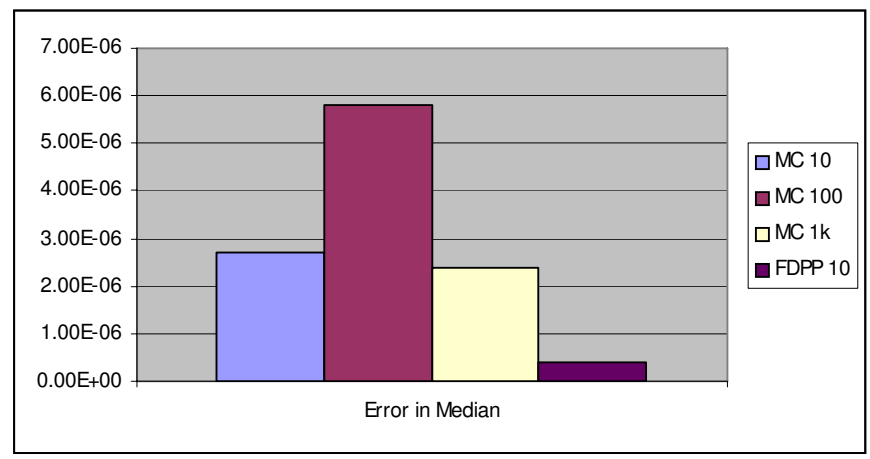

Figure 2. Error in median

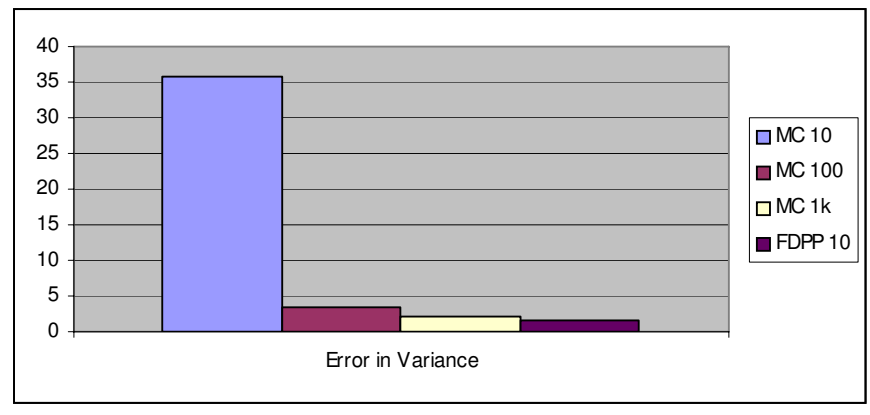

Figure 3. Error in variance

our benchmark. The behavioral simulator described in [22] is used for the analysis.

In support of our proposal, we have conducted a set of experiments centered largely on a PLL architecture as presented in [22]. This architecture is shown in Figure 1. Matlab R12 was employed for mathematical evaluations, by incorporating a Perl script to calculate the rms jitter at the voltage controlled oscillator output [10]. For each sample, $3 \mathrm{E}+5$ simulations steps are run with a sample period of $1 \mathrm{E}-$ 11 s. Each sample took 10 seconds to simulate on a $1.44 \mathrm{GHz}$ desktop. Two experiments have been run, in one of which the output current of the charge pump and in the other, the output frequency of the loop filter are assumed to vary with a probability density function with a $\sigma$ of $10 \% .{ }^{1}$ These densities stem from mismatch or process variations in the charge pump or the loop filter and are to be extracted using transistor level block simulations through HSPICE Monte Carlo simulations or FDPP. ${ }^{2}$

FDPP starts with a forward operator on the density samples of the charge-pump output current. 10 samples have been used according to the error bounds given in [5]. ${ }^{3}$ The

\footnotetext{
${ }^{1}$ Note that sample generation is the bulk of the runtime, whereas the implemented post-processing algorithms consume only a couple of seconds for all the samples.

${ }^{2}$ Running Monte Carlo simulations in the block level does not take significant time for most blocks when compared to the system simulation.

${ }^{3}$ The sample number selection in the re-bin operator is not automated but is given to the user as a choice and a theoretic limit because of similar issues in most mesh generation algorithms. Accordingly, one way to vali-
}

application of the forward operation is followed by a re-bin operation which re-bins the 10 samples.

Monte Carlo runs for the same example consume long run-times for the same accuracy. An accuracy comparison is shown in Figure 2 for median and in Figure 3 for variance. The error rates are given as percentages. Since we do not know the true density for the output jitter, a Monte Carlo run with 10k samples is assumed to give the reference true density. In reality, a Monte Carlo run with a sample number approaching infinity in the limit would give the true density. But since this is practically unattainable, $10 \mathrm{k}$ runs are assumed to yield the true density.

Two criteria are compared in the error figures. These criteria were the median and variance of the final densities. These statistical figures give us a measure of the method being able to estimate the density accurately. $M C$ in the figures represents a Monte Carlo run and FDPP in the figures represents the proposed method. The numbers next to these letters give the number of samples run, which are also proportional to the actual run-time, hence can be used to compare the run-times of different methods.

We have particularly paid attention to a fair comparison. Monte Carlo samples are first binned into 10 bins, the same number of samples used as in FDPP. Then, a 500-point spline interpolation is used for all methods. The error rates between 1k sample run Monte Carlo and 10-sample FDPP run are quite close, yet the run-time of 10-sample FDPP run is 100 times less than a $1 \mathrm{k}$ run Monte Carlo. Furthermore, FDPP has better accuracy both for median and variance. ${ }^{4}$

\section{Summary}

Forward discrete probability propagation (FDPP) has been suggested as an alternative to the Monte Carlo method. FDPP requires a far smaller number of samples than Monte Carlo using weight propagation and deterministic sampling. We introduce herein FDPP for behavioral level fast and accurate performance estimation. Behavioral simulations are increasingly popular as large circuits with possible feedback loops take a long time to simulate. Re-binning algorithm has been used to implement the accurate performance estimation system that uses deterministic sampling and probability weight propagation as the basis. FDPP, using a recent PLL structure as an example, is shown to be highly efficient for behavioral-level performance estimation and simulation by showing improved accuracy and 100 times run-time speed-up as compared to a standard 1000 sample Monte Carlo.

date the correct selection is to repeat the calculations with finer bin widths and compare that the results are within an error range.

${ }^{4}$ Notice that accurate median calculation also necessitates an accurate density representation. 


\section{References}

[1] P. Arcioni, M. Bozzi, M. Bressan, G. Conciauro, and L. Perregrini. Fast optimization, tolerance analysis, and yield estimation of h-/e-plane waveguide components with irregular shapes. IEEE Transactions on Microwave Theory and Techniques, 52(1):319-328, 2004.

[2] B. Balzs, Szirmay-Kalos, and A. Gyrgy. Weighted importance sampling in shooting algorithms. In SCCG '03: Proceedings of the 19th spring conference on Computer graphics, pages 177-184, New York, NY, USA, 2003. ACM Press.

[3] C. M. Bishop. Neural Networks for Pattern Recognition. Oxford University Press, 1995.

[4] A. Demir, E. Liu, A. L. Sangiovanni-Vincentelli, and I. Vassiliou. Behavioral simulation techniques for phase/delaylocked systems. In Custom Integrated Circuits Conference, pages 453-456, 1994.

[5] L. Devroye and G. Lugosi. Bin width selection in multivariate histograms by the combinatorial method. Test, 13:129145,2004

[6] P. G. Drennan and C. C. McAndrew. Understanding mosfet mismatch for analog design. IEEE JSSC, 38(3):450-456, 2003.

[7] P. Glasserman. Monte Carlo Methods in Financial Engineering. Springer-Verlag, 2003.

[8] A. Graupner, W. Schwarz, and R. Schuffny. Statistical analysis of analog structures through variance calculation. Circuits and Systems I: Regular Papers, IEEE Transactions on, 49:1071-1078, 2002.

[9] C. Guardiani, S. Saxena, P. McNamara, P. Schumaker, and D. Coder. An asymptotically constant, linearly bounded methodology for the statistical simulation of analog circuits including component mismatch effects. In Design Automation Conference, pages 15-18, 2000.

[10] A. Hajimiri and T. Lee. A general theory of phase noise in electrical oscillators. IEEE JSSC, 33(2):179-194, 1998.

[11] M. Hinz, I. Konenkamp, and E.-H. Horneber. Behavioral modeling and simulation of phase-locked loops for $\mathrm{rf}$ front ends. In Midwest Symposium on Circuits and Systems, pages 194-197, 2000.

[12] S. Inohira, T. Shinmi, M. Nagata, T. Toyabe, and K. Iida. A statistical model including parameter matching for analog integrated circuits simulation. Computer-Aided Design of Integrated Circuits and Systems, IEEE Transactions on, 4:621-628, 1985.

[13] V. Khandelwal and A. Srivastava. A general framework for accurate statistical timing analysis considering correlations. In Design Automation Conference, pages 89-94, 2005.

[14] F. Liu, J. Flomenberg, D. Yasaratne, and S. Ozev. Hierarchical variance analysis for analog circuits based on graph modelling and correlation loop tracing. In Design Automation and Test in Europe, pages 126-131, 2005.

[15] W.-L. Loh. On latin-hypercube sampling. Annals of Statistics, 24:2058-2080, 5.

[16] D. J. C. Mackay. Introduction to Monte Carlo Methods. Kluwer Academic Press, 1997.

[17] C. Michael and M. Ismail. Statistical modeling of device mismatch for analog mos integrated circuits. IEEE JSSC, 27(2):154-166, 1992.
[18] A. Mounir, A. Mostafa, and M. Fikry. Automatic behavioural model calibration for efficient pll system verification. In Design Automation and Test in Europe, pages 280-285, 2003.

[19] S. Ozev and A. Orailoglu. Boosting the accuracy of analog test coverage computation through statistical tolerance analysis. In VLSI Test Symposium, pages 213-219, 2002.

[20] P. M. Pardalos et al. Handbook of Applied Optimization. Oxfor University Press, 2002.

[21] M. Pelgrom, A. Duinmaijer, and A. Welbers. Matching properties of mos transistors. IEEE JSSC, 24(5):1433-1439, 1989.

[22] M. Perrott. Fast and accurate behavioral simulation of fractional-n synthesizers and other pll/dll circuits. In Design Automation Conference, pages 498-503, 2002.

[23] M. Rencher. Analog statistical simulation. In Custom Integrated Circuits Conference, pages 29.2/1-29.2/4, 1991.

[24] K. Saab, N. Ben-Hamida, and B. Kaminska. Parametric fault simulation and test vector generation. In Design Automation and Test in Europe, pages 650-656, 2000.

[25] A. Saltelli et al. Sensitivity Analysis. John Wiley \& Sons publishers, 2000.

[26] M. Seeger. Gaussian processes for machine learning.

[27] A. Srivastava, S. Shah, K. Agarwal, D. Sylvester, D. Blaauw, and S. Director. Accurate and efficient gate-level parametric yield estimation considering correlated variations in leakage power and performance. In Design Automation Conference, pages 535-540, 2005.

[28] E. Sudderth, A. Ihler, W. Freeman, and A. Willsky. Nonparametric belief propagation. In Computer Vision and Pattern Recognition, pages 605-612, 2003.

[29] J. Swidzinski, D. Alexander, M. Qu, and M. Styblinski. A systematic approach to statistical simulation of complex analog integrated circuits. In Int. Workshop on Statistical Metrology, pages 86-89, 1997.

[30] R. O. Topaloglu and A. Orailoglu. On mismatch in the deep sub-micron era-from physics to circuits. In ASP-DAC, pages 62-67, 2004.

[31] R. O. Topaloglu and A. Orailoglu. Forward discrete probability propagation method for device performance characterization under process variations. In Proceedings of Asian and Southern Pacific Design Automation Conference, pages 220-223, 2005.

[32] H. Yoon, P. Variyam, A. Chatterjee, and N. Nagi. Hierarchical statistical inference model for specification based testing of analog circuits. In VLSI Test Symposium, pages 145-150, 1999.

[33] L. Zhang, W. Chen, Y. Hu, J. A. Gubner, and C. C.-P. Chen. Correlation-preserved non-gaussian statistical timing analysis with quadratic timing model. In Design Automation Conference, pages 83-88, 2005.

[34] S. Zhang, V. Wason, and K. Banerjee. A probabilistic framework to estimate full-chip subthreshold leakage power distribution considering within-die and die-to-die p-t-v variations. In Low Power Electronics and Design, pages 156161, 2004. 